\title{
EVOLUÇÃO DAS PACIENTES SUBMETIDAS A CIRURGIA DE MAMA EM DRENAGEM ASPIRATIVA
}

Luciana Puchalski Kalinke, Kátia Renata Antunes Kochla², Liliana Maria Labronici², Thais Lima ${ }^{4}$, Angelita Visentin 5 , Ricardo Testoni ${ }^{6}$

\begin{abstract}
RESUMO: Estudo exploratório descritivo, teve como objetivo identificar as principais dificuldades que a paciente apresenta ao manusear o sistema de drenagem aspirativa em domicílio. Foi realizado em um hospital de referência em oncologia da cidade de Curitiba, de setembro a novembro de 2008, com 43 pacientes submetidas à cirurgia de mama e que utilizaram o dreno suctor. A coleta de dados ocorreu por meio de entrevista individual e avaliação clínica do enfermeiro no primeiro retorno ambulatorial; os dados foram quantificados e analisados por frequência absoluta simples. Constatou-se que $61 \%$ referiram não apresentar dificuldades para manipular o dreno, entre essas, $42 \%$ tiveram seus drenos manipulados por familiares ou amigos. $\mathrm{O}$ autocuidado faz parte de todo o processo de recuperação das mulheres no pós-operatório. Neste sentido salientamos o papel do profissional de enfermagem nas orientações pós-cirúrgicas para reduzir os riscos de complicações e favorecer a recuperação.
\end{abstract}

PALAVRAS-CHAVE: Câncer de mama; Mastectomia; Cuidados de enfermagem.

\section{THE EVOLUTION OF BREAST SURGERY PATIENTS IN THE USE OF ASPIRATIVE DRAINS}

ABSTRACT: This exploratory, descriptive study aimed to identify the principal difficulties which patients experienced in handling the aspiration drainage system at home. The study was carried out in an oncology reference hospital in the city of Curitiba, Brazil, between September and November 2008, with 43 patients who had had breast surgery and who were using suction drains. Data collection was from individual interviews and the nurses' clinical evaluation of the patients' first post-operative check-up visit to their doctor. The data was quantified and analysed by simple absolute frequency. It was ascertained that $61 \%$ stated that they did not have difficulties in using the drain; of these people, $42 \%$ had their drains cared for by families or friends. Self-care is part of the process of the women's recuperation in the post-operative period. This being so, the study emphasizes the role of the nursing professional in giving post-surgery advice, to reduce the risk of complications and assist recuperation.

KEYWORDS: Breast cancer; Mastectomy; Nursing care.

\section{EVOLUCIÓN DE LAS PACIENTES SOMETIDAS A LA CIRUGIA DE MAMA EN DRENAJE ASPIRATIVA}

RESUMEN: Estudio exploratorio descriptivo, que tuvo como objetivo identificar las principales dificultades que el paciente presenta al manipular el sistema de drenaje aspirativa en domicilio. Fue realizado en un hospital de referencia en oncología de la ciudad de Curitiba, de septiembre a noviembre de 2008, con 43 pacientes sometidas a cirugia de mama y que utilizaron el dreno suctor. Los datos fueron recogidos por medio de entrevista individual y evaluación clínica del enfermero en primer retorno ambulatorial; los datos fueron cuantificados y analizados por frecuencia absoluta simple. Se ha constatado que $61 \%$ afirmaron no presentar dificultades para manipular el dreno, entre eses, $42 \%$ tuvieron sus drenos manipulados por familiares o amigos. El autocuidado hace parte de todo el proceso de recuperación de las mujeres en el posoperatorio. En este sentido, destacamos el papel del profesional de enfermería en las orientacciones poscirúgicas para reducir los riesgos de complicaciones y favorecer la recuperación.

PALABRAS-CLAVE: Cáncer de mama; Mastectomía; Cuidados de enfermería.

${ }^{1}$ Enfermeira. Doutora em Ciências da Saúde. Professora do Curso de Graduação da Universidade Federal do Paraná - UFPR. Membro do Grupo de Pesquisa em Saúde do Adulto - GEMSA.

${ }^{2}$ Enfermeira da Prefeitura Municipal de Araucária e do Curso de Graduação em Enfermagem da Universidade Positivo. Mestre em Enfermagem. Doutoranda em Enfermagem pelo Programa de Pós-Graduação em Enfermagem - PPGENF UFPR. Membro do GEMSA. ${ }^{3}$ Enfermeira. Doutora em Enfermagem. Professora do Curso de Graduação e do PPGENF UFPR. Líder do GEMSA.

${ }^{4}$ Enfermeira do Hospital Erasto Gaertner. Especialista em Enfermagem Oncológica.

${ }^{5}$ Enfermeira. Mestre em Enfermagem. Coordenadora do Curso de Graduação em Enfermagem das Faculdades Integradas do Brasil. ${ }^{6}$ Enfermeiro do Setor de Radioterapia do Hospital Erasto Gaertner. Mestrando pelo Programa de Pós-Graduação em Distúrbios da Comunicação da Universidade Tuiuti do Paraná. 


\section{INTRODUÇÃO}

O carcinoma mamário invasor é a segunda neoplasia mais incidente na população feminina no Brasil, com cerca de 48.930 casos novos diagnosticados a cada ano, o que significa que a cada 100.000 mulheres 52 apresentarão câncer de mama ${ }^{(1)}$.

O diagnóstico do câncer mamário demanda um tempo prolongado de tratamento, exames diagnósticos e de controle até chegar ao procedimento cirúrgico. A grande maioria das pacientes com este diagnóstico submete-se a abordagem cirúrgica, o que compromete a axila com finalidade prognóstica. A formação de seroma pós-mastectomia é consequência da lesão dos vasos linfáticos e sanguíneos durante a ressecção cirúrgica da mama e é considerada a complicação mais comum, todavia, pode interferir no processo de cicatrização da ferida operatória e na disfunção dos movimentos do ombro e antebraço ${ }^{(2)}$. Alguns fatores que podem contribuir para a formação e incidência do seroma são: mamas volumosas com bastante tecido adiposo, técnica de cirurgia empregada como nas que removem o músculo grande e pequeno peitoral, e pacientes com sobrepeso ${ }^{(3)}$.

Estudos relacionados às complicações no pósoperatório de mastectomia decorrentes da alta precoce indicam que esta conduta é segura e benéfica para a maioria das pacientes, e pode ser recomendada quando há suporte domiciliar adequado, boas condições de saúde e fácil acesso à equipe de saúde. Ressaltam, ainda, a necessidade de que orientações pré-operatórias sejam fornecidas às pacientes e aos seus familiares ${ }^{(2,5-6)}$.

A enfermeira tem por atribuição ensinar a paciente e os membros da família a controlar o sistema de drenagem, principalmente para evitar as possíveis complicações, que podem incluir o acúmulo de sangue no sítio de incisão (hematoma), infecção e acúmulo tardio de líquido serossanguinolento (seroma) depois da remoção do dreno $^{(7)}$.

No hospital onde a presente pesquisa foi desenvolvida, as pacientes submetidas à mastectomia recebem alta hospitalar, mantendo o sistema de drenagem aspirativa em domicílio. Observa-se que a utilização desta rotina, quando corretamente aplicada, diminui o tempo de internação de cinco para dois dias ${ }^{(8)}$. O risco de formação de seroma também diminui, vez que o sistema de drenagem é retirado somente no primeiro retorno ambulatorial, entre o quinto e sétimo dia pós-cirúrgico.

Diante do exposto, a pesquisa teve como objetivo identificar as principais dificuldades que a paciente em pós-operatório apresenta ao manusear o sistema de drenagem aspirativa no domicílio, bem como, avaliar se esta rotina diminui as possíveis complicações e intercorrência.

\section{MATERIAL E MÉTODOS}

Trata-se de pesquisa exploratória descritiva, desenvolvida em um hospital de referência do sul do Brasil no tratamento do câncer, que está localizado no município de Curitiba - PR. É uma instituição filantrópica que atende cerca de 17.000 casos novos de câncer ao ano, e aproximadamente $80 \%$ dos atendimentos são pelo Sistema Único de Saúde (SUS).

Participaram da pesquisa 43 mulheres submetidas à cirurgia de mama e que utilizaram o dreno de suctor. Foram excluídas as que realizaram apenas biópsia e aquelas que não colocaram o dispositivo.

A coleta de dados ocorreu por meio de entrevista individual e avaliação clínica pelo enfermeiro no primeiro retorno ambulatorial destas pacientes, no período do estudo (setembro a novembro de 2008). Utilizou-se um instrumento norteador contendo as seguintes informações: perfil epidemiológico, dados cirúrgicos, orientações recebidas pelas pacientes e evolução no pós-operatório. Após a tabulação dos dados, os mesmos foram quantificados e analisados através de frequência absoluta simples.

A pesquisa foi aprovada pelo Comitê de Ética em Pesquisa com Seres Humanos do hospital (protocolo n. PP1645), e seu início ocorreu após a assinatura do Termo de Consentimento Livre e Esclarecido das participantes.

\section{RESULTADOS}

\section{Caracterização dos sujeitos do estudo}

Das pacientes, 40 estavam participando pela primeira vez de um procedimento cirúrgico, três já haviam feito cirurgia na mama, das quais duas realizaram nodulectomia e uma mastectomia. Quanto às cirurgias realizadas, $57,7 \%$ foram mastectomia radical modificada, e 31,8\% realizaram quadrantectomia com esvaziamento axilar.

Em relação à localização dos drenos, $62,2 \%$ estavam na axila direita, e $37,8 \%$ na axila esquerda. Todas as pacientes participantes da pesquisa permaneceram somente dois dias internadas.

Quanto ao perfil epidemiológico destacou-se a 
média de idade das pacientes era de 58,9 anos; quanto a escolaridade, no gráfico 1 , observa-se que $51,1 \%$ possuíam o ensino fundamental incompleto. Na atividade ocupacional exercida pelas pacientes, no gráfico 2, a atividade doméstica "do lar" ocupa a primeira colocação com, $51,1 \%$.

Gráfico 1 - Grau de escolaridade de mulheres submetidas a cirurgia de mama e uso de dreno. Curitiba, 2008

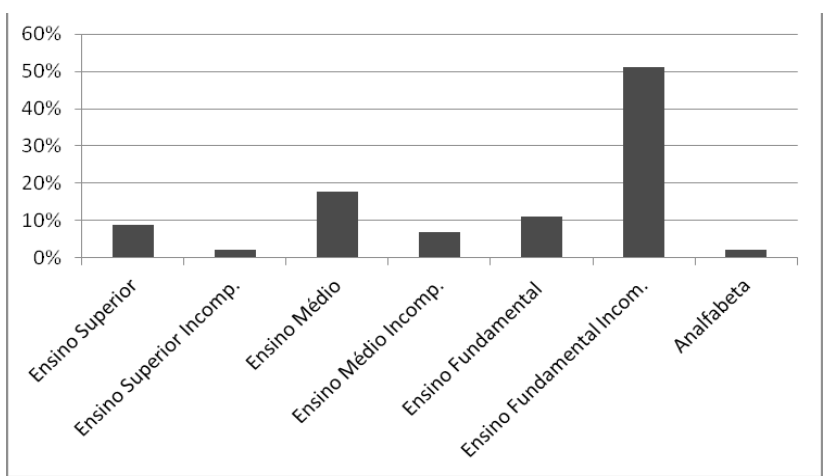

Gráfico 2 - Atividade ocupacional de mulheres submetidas a cirurgia de mama e uso de dreno. Curitiba, 2008

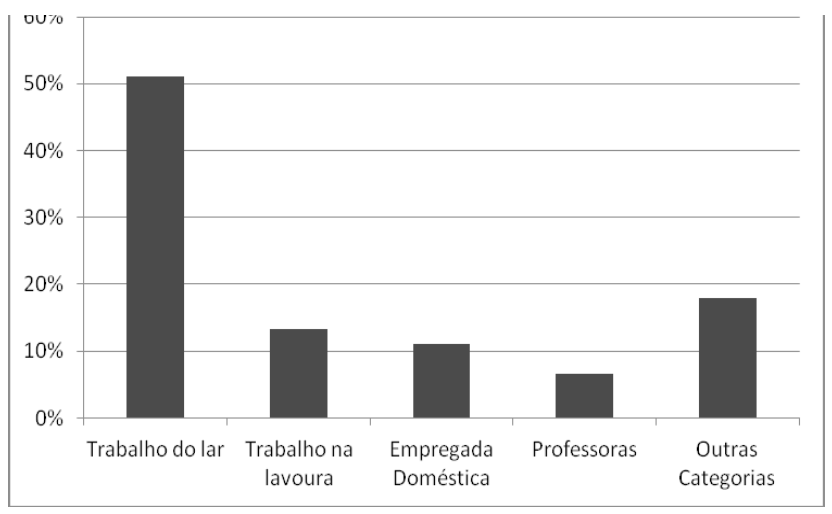

\section{Orientações recebidas na fase pré operatório}

Quando abordadas em relação à orientações recebidas antes das cirurgias relativas aos cuidados domiciliares, $84,4 \%$ tinham sido orientadas, $11,3 \%$ não receberam orientação e $0,5 \%$ não lembravam se haviam recebido ou não informações no período pré-operatório.

Em relação ao tipo de orientação recebida, 54,4\% mencionaram informações sobre o dreno e a cirurgia, $20 \%$ apenas sobre a cirurgia, $8,8 \%$ não sabiam dizer, e as demais não lembravam. A informação sobre a utilização do dreno foi recebida por $60 \%$, e $40 \%$ afirmaram não ter recebido nenhuma informação sobre a necessidade de sua utilização ou manejo.

A importância de ser informada sobre o uso de dreno antes da cirurgia, bem como sua utilização foi constatada em $79 \%$ das pacientes que acham importante esta orientação, e $21 \%$ não a consideraram relevante.

\section{Cuidados com o manuseio do dreno}

As pacientes que receberam orientações quanto ao manuseio do dreno foram questionadas sobre qual profissional as orientou, e $72 \%$ mencionaram que foi o médico, 9,3\% receberam informações da enfermeira, $13,9 \%$ não souberam responder, e $4,6 \%$ responderam que tanto o médico como a enfermeira explicitaram sobre a sua utilização.

Em relação à interferência da utilização do dreno nas atividades diárias, $74 \%$ relataram que houve interferência e 26\% afirmaram que não houve. Entre os relatos, observa-se:

\section{Dificuldade para dormir [...]. (Paciente 2)}

Não dava para fazer quase nada [...]. (Paciente 12)

Não consegui fazer nenhuma atividade [...]. (Paciente 22)

\section{[...]para tomar banho. (Paciente 28)}

\section{[...]para se movimentar. (Paciente 33)}

\section{[...]para trabalhar e para cozinhar. (Paciente 38)}

Quanto às dificuldades para manusear o dreno, $61 \%$ disseram que não tiveram, mas deste total $42 \%$ tiveram o dreno manipulado por outra pessoa. Entre as 39\% que referiram dificuldades, a totalidade relacionou ao esvaziamento do dispositivo.

\section{Evolução durante o pós operatório}

Entre as intercorrências apresentadas no período pós-operatório, a mais frequente foi o hematoma. Entre as 43 pacientes, apenas uma apresentou deiscência de sutura com secreção purulenta; as demais apresentaram pele limpa e seca e pontos íntegros, 8 apresentaram hematoma, duas seroma, duas hiperemia no local do dreno e duas tiveram dor e edema.

\section{DISCUSSÃO}

O câncer é reconhecido como uma doença crônicodegenerativa que atinge milhões de pessoas em todo o 
mundo. Apesar dos avanços terapêuticos permitirem melhoria na taxa de sobrevida, ainda permanece o estigma de doença incapacitante, mutiladora e mortal. Dessa forma, fica clara a necessidade e a propriedade de intervenções de enfermagem que auxiliem as pessoas no enfrentamento da doença e suas consequências, visando a reabilitação e a melhoria da qualidade de $\operatorname{vida}^{(9)}$.

Nas características epidemiológicas das pacientes observa-se que foram ao encontro do padrão de comportamento que a literatura traz sobre o perfil das mulheres com câncer de mama no Brasil: a média de idade de diagnóstico e mortalidade é entre 50 a 69 $\operatorname{anos}^{(10)}$, a baixa escolaridade apresenta menos oportunidade de diagnóstico precoce a estas pacientes ${ }^{(11)}$, a ocupação da mulher ainda tem a grande predominância de atividades domésticas.

A demora para o retorno das atividades domésticas gera ansiedade nas mulheres, visto que querem realizálas rapidamente, e isso traz a visão utilitarista do corpo, percebido como um instrumento para a maternidade, trabalho e manutenção da subsistência, e o adoecer é sinônimo de fraqueza, de incapacidade de auto-sustentação( ${ }^{(6)}$.

A mulher que desenvolve as atividades domésticas no lar, muitas vezes tem a preocupação da manutenção da família, pois é a responsável pelas atividades domésticas, alimentação, filhos e organização geral. Assim, a preocupação não se centra apenas no seu processo de adoecimento, mas em tudo que poderá deixar de ser feito em função da sua limitação temporária.

Quando ela adoece, existe a preocupação da doença e com todas as outras atividades que ficarão descobertas, portanto a reabilitação precoce é um elemento importante neste contexto.

No que diz respeito à média de dias de internação, as mulheres participantes da pesquisa receberam alta no segundo dia de pós-operatório. A alta precoce contribui para a diminuição do risco de infecção hospitalar, permite uma economia de custos de internação, e possibilita a reintegração mais rápida da paciente no contexto familiar ${ }^{(7)}$. Contudo, é fundamental que a enfermeira desenvolva ações educativas direcionadas para o autocuidado de maneira segura e tranquila.

Os resultados do presente estudo demonstram que a grande maioria das pacientes $(84,4 \%)$ recebeu orientação durante o pré-operatório, no entanto, esta orientação foi direcionada, principalmente, ao tipo de cirurgia que será realizada. Considerando-se que o profissional orientador foi o médico, este constiui-se em educador, e não o enfermeiro.

$\mathrm{Na}$ fase pré-operatória a assistência de enferma- gem permite esclarecer as mulheres sobre as técnicas invasivas as quais serão submetidas, bem como sua importância e desconfortos. É uma etapa indispensável ao atendimento, pois visa a prevenir ansiedade e o medo, permite-nos ter um cliente participativo e colaborativo com os procedimentos a serem realizados ${ }^{(6)}$.

Uma consulta de enfermagem no pré-operatório poderá suprir a necessidade de informações complementares e mais abrangentes, que podem ir desde a valorização e verbalização dos sentimentos das pacientes, até a identificação de possíveis potenciais de situações problema. Também permitirá a participação mais direta do enfermeiro dentro da equipe multidisciplinar.

A família também deverá ser incluída no processo de informação e esclarecimento, tanto em relação ao tratamento, quanto ao cuidado, porquanto representa um ponto de apoio fundamental para o crescimento interior da pessoa, sendo uma força positiva para as tomadas de decisões e, consequentemente, transformações de conceitos e comportamentos ${ }^{(12)}$.

É importante ressaltar que o adoecimento da mulher pode atingir toda estrutura familiar e, muitas vezes, com a necessidade de reorganização das atividades do cotidiano. Portanto, o enfermeiro deverá incluí-la em seu plano de cuidados e orientações, a fim de suprir as dúvidas apresentadas e minimizar a ansiedade de todos.

O cuidado operatório inicia no momento da internação e se estende até o domicílio. Assim, no cuidado domiciliar as ações de cuidados devem estar direcionadas para a manipulação correta do dreno, realização de curativos, e para as questões de educação em saúde. São necessários cuidados relacionados à informação no procedimento correto do manuseio dos drenos, na realização de curativos, nos cuidados em relação à educação em saúde bem como o apoio psicológico.

Quando o paciente não participa de seu cuidado durante o internamento, e apenas é ensinado a cuidar-se quando recebe alta hospitalar, muitas vezes a ansiedade se sobrepõe à razão, devido à pressa que o paciente tem de ir embora, ocasionando dúvidas e complicações que poderiam ser esclarecidas e evitadas com uma orientação eficaz no momento adequado.

A relação enfermeiro-paciente tem a finalidade de identificar e atender as necessidades de saúde do paciente e contribuir para melhorar a prática de enfermagem, ao criar oportunidade de aprendizagem, e despertar nos pacientes sentimentos de confiança, permitindo que eles se sintam satisfeitos e seguros ${ }^{(13)}$.

Os resultados da pesquisa demonstram que $61 \%$ 
das pacientes não tiveram dúvidas em relação ao manuseio do dreno, porém, destas, $42 \%$ não manusearam seu dreno. Para a prestação de uma assistência com qualidade em oncologia sugerimos que a enfermagem enfatize família como relevante no cuidado, visto que, no momento de uma doença de caráter maligno como o câncer, esta também fica afetada em sua integridade, podendo interferir na promoção de respostas adaptativas pelo cliente ${ }^{(12)}$.

$\mathrm{Na}$ instituição da pesquisa, o serviço de ginecologia e mama conta com uma enfermeira especialista em enfermagem oncológica que faz parte do serviço prestando assistência principalmente no período pósoperatório, o que permite atingir $100 \%$ das pacientes. Entretanto, a orientação de cuidados com o manuseio do dreno de sucção e curativo foi fornecida, majoritariamente, pelo médico; fato já relatado em outros trabalhos ${ }^{(5,14)}$. Talvez este processo aconteça devido ao profissional enfermeiro não fazer parte do processo de planejamento do tratamento do paciente, ou seja, estar atuante no período pré-operatório, durante o recebimento do diagnóstico do paciente. Tanto mulheres quanto seus familiares consideram a atuação do Enfermeiro como muito útil e eficiente, pois alivia a tensão, esclarece as dúvidas e ajuda o fortalecimento psicológico, permitindo que as mulheres enfrentem mais positivamente as adversidades surgidas com o diagnóstico da doença e seu tratamento ${ }^{(15)}$.

Em relação às intercorrências apresentadas no pós operatório, foi possível observar que uma paciente participante da pesquisa apresentou infecção. Em relação ao dreno $35 \%$ das pacientes tiveram obstrução e escape de ar, dificuldades comuns de serem apresentadas e fáceis de serem solucionadas. Ressalta-se que estas poderiam ser evitadas se o paciente tivesse mais conhecimento sobre o manuseio do dreno, e isto pode ser suprido no período de internamento da paciente, fazendo com que ela participe do seu autocuidado.

Os enfermeiros são responsáveis pela avaliação dos pacientes durante todo o processo de tratamento, para o entendimento dos cuidados a serem realizados por eles, demonstrando que estes cuidados serão de grande importância para a sua reabilitação ${ }^{(16-18)}$. Nesta perspectiva, percebemos que a mulher passa por um período perioperatório estressante e incerto, que gera medo do desconhecido. Assim, ressaltamos a importância do papel educativo na assistência e a inserção da cliente no processo decisório de seu tratamento, pois ela tem o direito de decidir, opinar e saber como vai cuidar de seu corpo ${ }^{(6)}$.

\section{CONSIDERAÇÕES FINAIS}

As doenças crônico-degenerativas, como o câncer, têm alcançado altos índices de prevalência entre as causas de morte no mundo, devendo os profissionais de saúde estar preparados para atuar nesta realidade.

No presente estudo foi possível observar que o enfermeiro não atua como educador de pacientes submetidos à cirurgia de mama, sendo este espaço ocupado pelo profissional médico. Ressalta-se a importância da participação do enfermeiro desde o primeiro momento que a paciente recebe a notícia de que será necessária uma cirurgia, fazendo com que participe de modo efetivo na recuperação e reabilitação precoces.

\section{REFERÊNCIAS}

1. Ministério da Saúde(BR). Instituto Nacional do Câncer Nacional - INCA. Estimativa de Câncer no Brasil. 2010 [acesso em 1 nov 2010]. Disponível: http://www.inca. gov.br/estimativa/2010/.

2. Panobianco MS, Parra MV, Almeida AM, Prado MAS, Magalhães PAP. Estudo da adesão as estratégias de prevenção e controle de linfedema em mastectomizadas. Esc Anna Nery. 2009;13(1):161-8.

3. Hernanz F, Regano S, Redondo-Figuero C, Orallo V, Erasun F, Gomez-Fleitas M. Oncoplastic breastconserving surgery: analysis of quadrantectomy and immediate reconstruction with latissimus dorsi flap. World J Surg. 2007;31(10):1934-40.

4. Gonzalez EA, Saltzstein EC, Riedner CS, Nelson BK. Seroma formation following breast cancer surgery. Breast J. 2003;9(5):385-8.

5. Wronska P, Stepien R, Dobrowolska B. Satisfaction of women after mastectomy for nursing care. Advances in Medical Sciences. 2007; Supp152:S34-6.

6. Barreto RAS, Suzuki K, Lima MA, Moreira AA. As necessidades de informações de mulheres mastectomizadas subsidiando a assistência de enfermagem. Rev Eletr Enf. 2008;10(1):110-23.

7. Gutierrez MGR, Pinelli FGS, Areias VL, Barbi T. Avaliação de um programa educativo sobre os cuidados com o sistema de drenagem para cuidadores de mulheres mastectomizadas. Acta Paul Enferm. 2004;17(4):412-8.

8. Bregagnoll RK, Dias AS. Alterações funcionais em mulheres submetidas à cirurgia de mama com 
linfadenectomia axilar total. Rev Bras Cancerol. 2010;56(1):25-36.

9. Batalha MMF, Camargo TC, dos Santos Guedes MT, Los de Alcantara LF. Cancer, poverty and human development: challenges for nursing care in oncology. Rev Latino-Am Enfermagem. 2007;15(n.esp):780-5.

10. Pinho VF, Coutinho ES. Risk factors for breast cancer: a systematic review of studies with female samples among the general population in Brazil. Cad Saúde Pública. 2005;21(2):351-60.

11. Guerra MR, Mendonça GAS. Risco de câncer no Brasil: tendências e estudos epidemiológicos mais recentes. Rev Bras Cancerol. 2005;51(3):227-34.

12. Melo EM, Silva RM, Fernandes AFC. O Relacionamento familiar após a mastectomia: um enfoque no modo de interdependência de Roy. Rev Bras Cancerol. 2005;51(3):219-25.

13. Oliveira PS, Nobrega MML, Silva ATM, Filha MOF. Comunicação terapêutica em enfermagem revelada nos depoimentos de pacientes internados em centro de terapia intensiva. Rev Eletr Enf. 2005;7(1):54-63.

14. Romagosa-Albacar C, Garatea-Zubieta A, Inoriza JM. Nursing care in women with breast disease: organization of a specific nurses' clinic. Enf Clin. 2007;17(1):37-40.

15. Camargo TC, Souza IE. Care to mastectomized woman: discussing ontic aspects and the ontological dimension in nurses performance at a Cancer Hospital. Rev LatinoAm Enfermagem. 2003;11(5):614-21.

16. Pompeo DA, Pinto MH, Cesarino CB, Araujo RRDF, Poletti NAA. Nurses' performance on hospital discharge: patients' Point of view. Acta Paul Enferm. 2006;20(3):345-50.

17. Maieski VM, Sarquis LMM. Mulheres com câncer de mama em quimioterapia e sua influência sobre o trabalho. Cogitare Enferm. 2007;12(3):246-52.

18. Zandonai AP, Cardozo FMC, Nieto ING, Sawada NO. Qualidade de vida nos pacientes oncológicos: revisão integrativa da literatura latino-americana. Rev Eletr Enf. 2010;12(3):54-63.

Cogitare Enferm. 2011 Out/Dez; 16(4):689-94 